\title{
Electron current to a probe in a magnetized, collisional plasma
}

\author{
M. Charro and J. R. Sanmartín ${ }^{\text {a) }}$ \\ Escuela Técnica Superior de Ingenieros Aeronáuticos, Universidad Politécnica de Madrid, \\ 28040, Madrid, Spain
}

(Received 29 November 1999; accepted 14 February 2000)

\begin{abstract}
A collisional analysis of electron collection by a probe in a strongly magnetized, fully ionized plasma is carried out. A solution to the complete set of macroscopic equations with classical transport coefficients that is wholly consistent in the domain $1 \ll R^{2} / l_{e \infty}^{2} \ll\left(m_{i} / m_{e}\right)^{3 / 2}$ is determined; $R$ and $l_{e \infty}$ are probe radius and electron gyroradius, respectively. If $R^{2} / l_{e \infty}^{2}$ is large compared with $m_{i} / 3 m_{e}$ (probe large compared with ion gyroradius), ion-electron energy exchange-rather than electron heat diffusion-keeps electrons isothermal. For smaller probes at negative bias, however, electron cooling occurs in the plasma beyond the sheath, with a potential overshoot lying well away from it. The probe characteristic in the electron-retarding range may then mimic the characteristic for a two electron-temperature plasma and lead to an overestimate of electron temperature; the validity of these results for other transport models is discussed. (C) 2000 American Institute of Physics. [S1070-664X(00)00206-8]
\end{abstract}

\section{INTRODUCTION}

There is no established theory of electron collection by probes in strongly magnetized plasmas. Difficulties arise from the ensuing strong anisotropy in charge transport (essential to the working of probes as particle sinks), and the associated fact that, no matter how small the Debye length $\lambda_{\text {De }}$, perturbations reach far from the probe. Basic collisionless ${ }^{1-3}$ and turbulent ${ }^{4-6}$ models do not describe how faraway current perturbations die off, and do no lead to definite predictions for electron current, while a purely collisional model may be too requiring as regards perturbation distances. $^{7-9}$

In addition, the variety of parameters involved in magnetized plasmas allow for quite different probe regimes. Collisionless analyses may consider highly positive probe bias, corresponding to possible uses in unbounded, cold, rarefied space plasmas ${ }^{10}$ with new and important applications of probe theory. ${ }^{11}$ Recent ionospheric experiments suggest that spacecraft velocity, though highly subsonic as regards electrons, may have a substantial effect on electron collection, ${ }^{12-14}$ and that electrons in the plasma beyond the sheath get hotter in the collection process. ${ }^{15-17}$ Collisional theories usually consider negative or slightly positive bias, as appropriate for bounded, hotter and denser laboratory plasmas. ${ }^{18}$ New types of Langmuir probes use the ion branch of the probe characteristic at the edge of strongly ionized fusion plasmas (Mach and flush mounted probes to measure ion flow ${ }^{5,19}$ and density, ${ }^{20,21}$ respectively) but the need to determine the electron temperature $T_{e \infty}$ remains. Unfortunately, there is no complete and satisfactory theory of electron collection. ${ }^{20}$

Here we reconsider the collisional model of a fully ionized plasma using a macroscopic description instead of the old kinetic description. ${ }^{7}$ The present approach is more cum-

${ }^{a)}$ Electronic mail: jrs@faia.upm.es bersome if complete but allows clarifying a point in the kinetic analysis that appears uncertain when reexamined; whether the electron collection process has an isothermal character. We prove here that a dimensionless length parameter determines electron behavior in this respect. Our analysis is shown to be valid for

$$
1 \ll\left(R / l_{e \infty}\right)^{2} \ll\left(m_{i} / m_{e}\right)^{3 / 2},
$$

where $l_{e \infty}$ is the electron thermal gyroradius and $R$ is the radius of a disc normal to the magnetic field $\bar{B}$ (or the projected circular area of other type of probe, say, a sphere).

Within this parametric domain we find that electrons do keep isothermal for

$$
m_{i} / 3 m_{e} \ll\left(R / l_{e \infty}\right)^{2},
$$

with ion-electron energy exchange, rather than electron heat diffusion, accounting for that condition. At lower $R / l_{e_{\infty}}$, electron cooling occurs below some value of probe bias $\Phi_{P}$; this might affect the interpretation of probe data. In Secs. II-IV we obtain a fully consistent description of the perturbations caused by the probe in a collision-dominated outer region; closing boundary conditions are established in Sec. V. In Sec. VI we discuss our results and suggest they might be applicable to other transport models.

\section{COLLISIONAL EQUATIONS}

We consider a fully ionized plasma in the presence of an uniform magnetic field along the $z$-axis of cylindrical coordinates $r, \theta$, and $z$. We assume a steady, collisional regime with strongly magnetized electrons, $\lambda_{e \infty} / l_{e \infty} \sim \Omega_{e} \tau_{e \infty} \gg 1$, where $\Omega_{e} \equiv e B / m_{e}$ is the electron gyrofrequency, and $\tau_{e \infty}$ and $\lambda_{e \infty}$ are unperturbed electron collision time and mean free path, respectively. We consider the electron current to a probe of radius $R \gg l_{e \infty}$ and look for a consistent solution to the complete set of macroscopic equations with classical transport coefficients. Dimensionless results in the solution 
may only depend on parameters $e \Phi_{P} / k T_{e \infty}, T_{i \infty} / T_{e \infty}$, and ion charge number $Z_{i}$, which we take of order unity, in addition to the large parameters $\lambda_{e \infty} / l_{e \infty}, R / l_{e \infty}$, and $m_{i} / m_{e}$. We take the relative ordering $\lambda_{e \infty} / l_{e \infty} \geqslant O\left(R / l_{e \infty}\right)$ and, for simplicity in dealing with ion transport, ions moderately magnetized at least, $\lambda_{e \infty} / l_{e \infty} \geqslant O\left(\sqrt{ } m_{i} / m_{e}\right) \rightarrow \lambda_{e \infty} / l_{i \infty}$ $\geqslant O(1)$. No sheath analysis is required, the quasineutrality condition being used throughout some outer region, and $\lambda_{\text {De }}(\ll R)$ actually not entering the results.

The steady continuity, momentum and energy equations for ions or electrons $(\alpha=i, e)$ read

$$
\nabla \cdot n_{\alpha} \bar{v}_{\alpha}=0
$$

$m_{\alpha} n_{\alpha} \bar{v}_{\alpha} \cdot \nabla \bar{v}_{\alpha}=-\nabla p_{\alpha}-\nabla \cdot \overline{\bar{\Pi}}_{\alpha}+e_{\alpha} n_{\alpha}\left(\bar{E}+\bar{v}_{\alpha} \wedge \bar{B}\right)+\bar{R}_{\alpha}$,

$\nabla \cdot\left[\left(\frac{m_{\alpha} v_{\alpha}^{2}}{2}+\frac{5}{2} k T_{\alpha}+e_{\alpha} \Phi\right) n_{\alpha} \bar{v}_{\alpha}+\bar{q}_{\alpha}+\bar{\Pi}_{\alpha} \cdot \bar{v}_{\alpha}\right]$

$$
=\bar{R}_{\alpha} \cdot \bar{v}_{\alpha}+Q_{\alpha},
$$

where we have $e_{i} / Z_{i}=-e_{e} \equiv e, \bar{E}=-\nabla \Phi, p_{\alpha}=n_{\alpha} k T_{\alpha}$, $\partial / \partial \theta \equiv 0$, and $Z_{i} n_{i} \approx n_{e} \equiv n$ (quasineutrality). The stress tensor in the viscous force $\bar{F}_{v \alpha} \equiv-\nabla \cdot \bar{\Pi}_{\alpha}$, given by Braginskii, ${ }^{22}$ involves five viscous coefficients $\left(\eta_{0}-\eta_{4}\right)$ for each species. ${ }^{23}$

The ion-electron force, $-\bar{R}_{i}=\bar{R}_{e} \equiv \bar{R}$, has a tensor character, and is made of terms linear in either $\bar{u} \equiv \bar{v}_{e}-\bar{v}_{i}$ (Hall and nonisotropic-Ohm effects) or $\nabla T_{e}$ (thermoelectric Nernst and nonisotropic-Seebeck effects). One also has

$$
\bar{R}_{e} \cdot \bar{v}_{e}+Q_{e}=-\bar{R}_{i} \cdot \bar{v}_{i}-Q_{i},
$$

with $\mathrm{Q}_{i} \propto\left(T_{e}-T_{i}\right)$. The electron heat flux $\bar{q}_{e}$ is again made of terms linear in either $\nabla T_{e}$ (Righi-Leduc and nonisotropicFourier terms) or $\bar{u}$ (thermoelectric Ettinghausen and nonisotropic-Peltier terms), whereas $\bar{q}_{i}$ only involves terms linear in $\nabla T_{i}$. All coefficients are given by Braginskii ${ }^{22}$ (with some corrections by Epperlein and Haines ${ }^{24}$ ). The formalism in Ref. 22 proves far more convenient than alternative transport formalisms. ${ }^{25}$

\section{MODEL REGIME}

In solving Eqs. (1)-(3) we make several ansatzen, shown to hold within some parametric domain in the next section. We take as negligible (i) $m_{\alpha} v_{\alpha}^{2}$ against $k T_{\alpha}$; (ii) $\nabla T_{i}$; (iii) $F_{\text {vir }}$; (iv) $\bar{v}_{i}$ against $\bar{v}_{e}$; (v) $R_{r}$; (vi) the electron viscous stress tensor $\bar{\Pi}_{e}$; and (vii) $\bar{R} \cdot \bar{v}_{i}$ against $Q_{i}$. We can then separately solve the ion $r$-momentum equation in (2) and all three Equations (1)-(3) for electrons, which become uncoupled from the remaining equations. Taking into account ansatzen (i) $-(\mathrm{v})$ the first equation reads

$$
\frac{k T_{i \infty}}{Z_{i}} \frac{\partial n}{\partial r}=-e n \frac{\partial \Phi}{\partial r} .
$$

Using the boundary conditions $n \rightarrow n_{\infty}, \Phi \rightarrow 0$, as $r \rightarrow \infty$ at fixed $z$, Eq. (5) gives

$$
n(r, z)=n_{\infty} \exp \left(\frac{-Z_{i} e \Phi(r, z)}{k T_{i \infty}}\right) .
$$

The electron momentum equations read

$$
\begin{aligned}
& \frac{\partial p_{e}}{\partial z}-e n \frac{\partial \Phi}{\partial z}=R_{z} \rightarrow-\alpha_{0}\left(Z_{i}\right) \frac{m_{e} n}{\tau_{e}} v_{e z}-\beta_{0}\left(Z_{i}\right) n k \frac{\partial T_{e}}{\partial z} \\
& -e B n v_{e r}=R_{\theta} \rightarrow-\frac{m_{e} n}{\tau_{e}} v_{e \theta}-\frac{\beta_{1}^{\prime \prime}}{\Omega_{e} \tau_{e}} n k \frac{\partial T_{e}}{\partial r} \\
& e B n v_{e \theta}=-\frac{\partial p_{e}}{\partial r}+e n \frac{\partial \Phi}{\partial r}
\end{aligned}
$$

In Eqs. (6) and (7) we used ansatzen (i), (iv), and (vi); in Eq. (8) we used ansatzen (i), (v), and (vi). In Eq. (6), $\alpha_{0}$ and $\beta_{0}$ are $\mathrm{Ohm}$ and Seebeck coefficients given by Braginskii. ${ }^{22} \mathrm{On}$ the right-hand side of (7) there are only Ohm and Nernst terms (with $\beta_{1}{ }^{\prime}{ }^{\prime}=3 / 2$ ); there is no Seebeck term because $\partial T_{e} / \partial \theta=0$, while the collisional Hall effect ends out to be smaller than the Ohm effect by a factor of order $\left(l_{e \infty} / \lambda_{e \infty}\right)^{5 / 3}$ (we used corrections to Braginskii's results from Ref. 24). The electron collision time, given in Ref. 22, takes the local value $\tau_{e}=\tau_{e \infty} T_{e}^{3 / 2} n_{\infty} / T_{e \infty}^{3 / 2} n$.

Equations $\left(5^{\prime}\right),(6)-(8)$ are now used to eliminate $n$ and $n v_{e \theta}$ and obtain the particle fluxes $n v_{e z}$ and $n v_{e r}$ in terms of $\Phi, T_{e}$, and their $z$ and $r$ gradients. The continuity equation (1) for electrons then provides a first equation relating $\Phi$ (or $n)$ and $T_{e}$. Introducing dimensionless variables,

$$
\begin{aligned}
& \tilde{r} \equiv \frac{r}{R}, \quad \tilde{z} \equiv \frac{z}{L_{z}}, \quad\left(L_{z} \equiv \frac{R \Omega_{e} \tau_{e \infty}}{\sqrt{\alpha_{0}}}\right), \\
& -2 \ln \left(\frac{n}{n_{\infty}}\right)=\widetilde{\Phi} \equiv \frac{2 Z_{i} e \Phi}{k T_{i \infty}}, \quad \widetilde{T}_{e} \equiv \frac{T_{e}}{T_{e \infty}}, \quad \widetilde{T}_{i \infty} \equiv \frac{T_{i \infty}}{T_{e \infty}},
\end{aligned}
$$

the continuity equation reads

$$
\begin{aligned}
& \frac{\partial}{\partial \tilde{z}}\left[\widetilde{T}_{e}^{3 / 2}\left\{\left(\widetilde{T}_{e}+\frac{\widetilde{T}_{i \infty}}{Z_{i}}\right) \frac{\partial \widetilde{\Phi}}{\partial \tilde{z}}-2\left(1+\beta_{0}\right) \frac{\partial \widetilde{T}_{e}}{\partial \tilde{z}}\right\}\right] \\
& \quad+\frac{1}{\tilde{r}} \frac{\partial}{\partial \tilde{r}}\left[\tilde{r} \frac{\exp (-\widetilde{\Phi})}{\widetilde{T}_{e}^{3 / 2}}\left\{\left(\widetilde{T}_{e}+\frac{\widetilde{T}_{i \infty}}{Z_{i}}\right) \frac{\partial \widetilde{\Phi}}{\partial \tilde{r}}+\frac{\partial \widetilde{T}_{e}}{\partial \tilde{r}}\right\}\right]=0 .
\end{aligned}
$$

Next, the energy equation for electrons, using Eq. (4) and ansatzen (i), (vi), and (vii), becomes

$$
\nabla \cdot\left[\left(\frac{5}{2} k T_{e}-e \Phi\right) n \bar{v}_{e}+\bar{q}_{e}\right]=-Q_{i} \equiv-\frac{3 m_{e}}{m_{i}} n k \frac{T_{e}-T_{i}}{\tau_{e}},
$$

with

$$
\begin{aligned}
& q_{e z}=-\gamma_{0}\left(Z_{i}\right) \frac{\tau_{e}}{m_{e}} n k T_{e} k \frac{\partial T_{e}}{\partial z}+\beta_{0} n k T_{e} v_{e z}, \\
& q_{e r}=-\frac{\gamma_{1}^{\prime}\left(Z_{i}\right)}{\Omega_{e}^{2} \tau_{e}^{2}} \frac{\tau_{e}}{m_{e}} n k T_{e} k \frac{\partial T_{e}}{\partial r}-\frac{\beta_{1}^{\prime \prime}}{\Omega_{e} \tau_{e}} n k T_{e} v_{e \theta} .
\end{aligned}
$$

Here, $\gamma_{0}$ and $\gamma_{1}$ ' are Fourier coefficients, while $\beta_{0}$ is now a Peltier coefficient. Only Fourier and Ettinghausen $\left(\beta_{1}{ }^{\prime}\right.$ ') terms appear in (14); there is no Righi-Leduc term because 
$\partial T_{e} / \partial \theta=0$, while the Peltier effect is smaller than the Ettinghausen effect by a factor $\left(l_{e \infty} / \lambda_{e \infty}\right)^{5 / 3}$. Taking $n, n v_{e z}$, and $n v_{e r}$ from Eqs. $\left(5^{\prime}\right)$, and (6)-(8), and again using dimensionless variables, (12) becomes

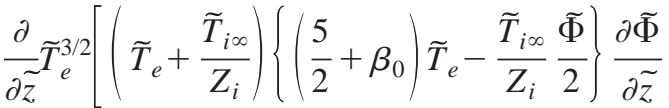

$$
\begin{aligned}
& \left.-\left\{2\left(\alpha_{0} \gamma_{0}+\frac{5}{2}+\frac{7}{2} \beta_{0}+\beta_{0}^{2}\right) \widetilde{T}_{e}-\left(1+\beta_{0}\right) \frac{\widetilde{T}_{i \infty}}{Z_{i}} \widetilde{\Phi}\right\} \frac{\partial \widetilde{T}_{e}}{\partial \tilde{z}}\right] \\
& +\frac{1}{\tilde{r}} \frac{\partial}{\partial \tilde{r}} \tilde{r} \frac{\exp (-\widetilde{\Phi})}{\widetilde{T}_{e}^{3 / 2}}\left[\left(\widetilde{T}_{e}+\frac{\widetilde{T}_{i \infty}}{Z_{i}}\right)\left(\widetilde{T}_{e}-\frac{\widetilde{T}_{i \infty}}{Z_{i}} \frac{\widetilde{\Phi}}{2}\right) \frac{\partial \widetilde{\Phi}}{\partial \tilde{r}}\right. \\
& \left.+\left\{\left(\frac{11}{2}-2 \gamma_{1}^{\prime}\right) \widetilde{T}_{e}-\frac{\widetilde{T}_{i \infty}}{Z_{i}} \frac{\widetilde{\Phi}}{2}\right\} \frac{\partial \widetilde{T}_{e}}{\partial \tilde{r}}\right] \\
& =-\frac{3 m_{e} R^{2}}{m_{i} l_{e^{\infty}}^{2}} \frac{\exp (-\widetilde{\Phi})}{\widetilde{T}_{e}^{3 / 2}}\left(\widetilde{T}_{e}-\widetilde{T}_{i \infty}\right) .
\end{aligned}
$$

In Ref. $7, T_{e}$ was considered uniform, thus ignoring Eq. (12') and simplifying Eq. (11).

\section{VALIDITY OF THE MODEL}

The analysis leading to Eq. (11) provides values for the characteristic length along $z$, and for all components of the electron velocity,

$$
\begin{aligned}
& L_{z} \sim R \Omega_{e} \tau_{e \infty} \sim \lambda_{e \infty} \frac{R}{l_{e \infty}}, \\
& v_{e z} \sim v_{e \theta} \sim \frac{\lambda_{e \infty}}{l_{e \infty}} v_{e r} \sim \frac{l_{e \infty}}{R} \times \text { electron thermal velocity. }
\end{aligned}
$$

These results may be now used to verify the ansatzen of the last section. As advanced in Sec. II, in the analysis of ion transport we assume that ions are at least moderately magnetized,

$$
\frac{\lambda_{e \infty}}{l_{i^{\infty}}} \geqslant O(1) \rightarrow \frac{\lambda_{e \infty}}{l_{e^{\infty}}} \geqslant O\left(\sqrt{\frac{m_{i}}{m_{e}}}\right) .
$$

Under ansatzen (i), (iv), and Eq. $\left(5^{\prime}\right)$, the two missing ionmomentum equations become

$$
\begin{aligned}
& R_{z}=F_{v i z} \rightarrow \frac{1}{r} \frac{\partial}{\partial r}\left(r \eta_{2} \frac{\partial v_{i z}}{\partial r}\right), \\
& R_{\theta}=F_{v i \theta} \rightarrow \frac{1}{r} \frac{\partial}{\partial r}\left(r \eta_{1} \frac{\partial v_{i \theta}}{\partial r}\right) .
\end{aligned}
$$

In the viscous force $F_{v i z}$ we find that $\eta_{0}$ and $\eta_{4}$ terms are smaller than the dominant $\eta_{2}$ term by factors of order $m_{e} / m_{i}$ and $\left(l_{e \infty} / \lambda_{e \infty}\right) \sqrt{ } m_{e} / m_{i}$, respectively, while $\eta_{1}$ and $\eta_{3}$ terms vanish; in $F_{v i \theta}, \eta_{0}$, and $\eta_{2}$ terms vanish, and $\eta_{3}$ and $\eta_{4}$ terms are small by a factor $\sqrt{ } m_{e} / m_{i} \cdot{ }^{22}$

Equations (18) and (19) lead to characteristic values for $v_{i z}$ and $v_{i \theta}$ when Eqs. (6) and (7) are used. The continuity equation (1) for ions then yields a characteristic value for $v_{i r}$. Using now Eq. (16) allows comparing ion and electron velocities,

$$
\frac{v_{i z}}{v_{e z}} \sim \frac{v_{i \theta}}{v_{e \theta}} \sim \frac{v_{i r}}{v_{e r}} \sim \frac{R^{2}}{l_{e \infty}^{2}}\left(\frac{m_{e}}{m_{i}}\right)^{3 / 2},
$$

and determining characteristic values for all inertia terms in the momentum equations,

$$
\begin{aligned}
& \frac{e-\text { inertia terms }}{\text { dominant terms }} \sim \frac{l_{e \infty}^{2}}{R^{2}}, \\
& \frac{i \text {-inertia terms }}{\text { dominant terms }} \sim \frac{R^{2}}{l_{e^{\infty}}^{2}}\left(\frac{m_{e}}{m_{i}}\right)^{2} .
\end{aligned}
$$

We can also determine a characteristic value for $R_{r}$,

$$
\frac{\text { Ohm, Seebeck and collisional Hall } R_{r} \text {-terms }}{\text { terms in Eqs. }(5,8)} \sim \frac{l_{e^{\infty}}^{2}}{\lambda_{e^{\infty}}^{2}} ;
$$

again, there is no Nernst term in $R_{r}$ because $\partial T_{e} / \partial \theta=0$. We then obtain

$$
\frac{\bar{R} \cdot \bar{v}_{i}}{Q_{i}} \sim \frac{R^{2}}{l_{e^{\infty}}^{2}}\left(\frac{m_{e}}{m_{i}}\right)^{3 / 2} .
$$

We next find

$$
\frac{F_{v i r^{-}} \text {term }}{\text { terms in Eq.(5) }} \sim \sqrt{\frac{m_{e}}{m_{i}}},
$$

where

$$
F_{v i r}=\frac{1}{r} \frac{\partial}{\partial r}\left(r \eta_{3} \frac{\partial v_{i \theta}}{\partial r}\right)
$$

the $\eta_{4}$ term in $F_{v i r}$ vanishes, the $\eta_{0}$ term is small by a factor ${ } m_{e} / m_{i}$, and $\eta_{1}$ and $\eta_{2}$ terms are small by a factor of order $l_{e \infty} / \lambda_{e \infty}$ at most. ${ }^{22}$ Concerning the electron viscous terms we find

$$
\begin{gathered}
\frac{F_{v e z}}{F_{v i z}} \sim \frac{F_{v e \theta}}{F_{v i \theta}} \sim \frac{F_{v e r}}{\partial p_{e} / \partial r} \sim \frac{l_{e \infty}^{2}}{R^{2}}, \\
\frac{\bar{\Pi}_{e}-\text { terms }}{p_{e} \text { term in Eq. (3) }} \sim \frac{l_{e^{\infty}}^{2}}{R^{2}} .
\end{gathered}
$$

Finally, we note that the dominant term in Eq. (3) for ions is that part of the heat-flux divergence arising from the radial flux,

$$
0 \approx-\frac{1}{r} \frac{\partial}{\partial r} r q_{i r},\left(q_{i r} \propto \frac{\partial T_{i}}{\partial r}\right) \rightarrow T_{i}(r, z) \approx T_{i \infty},
$$

with a small correction arising from the $Q_{i}$ term,

$$
\frac{Q_{i}}{\nabla \cdot \bar{q}_{i}} \sim \frac{R^{2}}{l_{e \infty}^{2}}\left(\frac{m_{e}}{m_{i}}\right)^{3 / 2} .
$$

Relations (20)-(26) concerning ansatzen (i)-(vii) in Sec. III show that our model applies for conditions

$$
1 \ll \frac{R^{2}}{l_{e^{\infty}}^{2}} \ll\left(\frac{m_{i}}{m_{e}}\right)^{3 / 2},
$$




$$
1 \ll \frac{\lambda_{e \infty}^{2}}{l_{e \infty}^{2}}, \quad\left(\Omega_{e}^{2} \tau_{e^{\infty}}^{2} \gg 1\right) .
$$

Note that our simplifying assumption (17) is more stringent than condition $\left(17^{\prime}\right)$.

\section{MODEL CLOSURE}

Equations (11) and $\left(12^{\prime}\right)$ determine $\Phi$ and $T_{e}$ when boundary conditions are given. Only one half-space, say $z$ $>0$, needs to be considered. Obvious conditions (written in dimensionless variables) are then

$$
\begin{aligned}
& \widetilde{\Phi} \rightarrow 0, \widetilde{T}_{e} \rightarrow 1 \quad \text { as } \tilde{z} \rightarrow \infty \text { or } \tilde{r} \rightarrow \infty, \\
& \frac{\partial \widetilde{\Phi}}{\partial \tilde{r}}=\frac{\partial \widetilde{T}_{e}}{\partial \tilde{r}}=0 \quad \text { at } \tilde{r}=0, \\
& \frac{\partial \widetilde{\Phi}}{\partial \tilde{z}}=\frac{\partial \widetilde{T}_{e}}{\partial \tilde{z}}=0 \quad \text { at } \tilde{z}=0, \tilde{r}>1 .
\end{aligned}
$$

Conditions at $\tilde{z}=0, \tilde{r}<1$ require detailed consideration. ${ }^{7}$

Equations (11) and $\left(12^{\prime}\right)$ describe an outer flow with collision-dominated electrons having density given by $\left(5^{\prime}\right)$, as a result of both quasineutrality and the fact that dominant radial gradients make ions follow a Boltzmann law. All this fails in some inner region adjoining the probe, with $\Phi_{0} \neq \Phi_{P}$ in particular; we shall use subscript 0 for values at $\tilde{z}=0, \tilde{r}$ $<1$. The ratio $n_{\infty} / n_{0}$, though possibly large, will be shown to be small compared with $R / l_{e \infty}$. As $\tilde{z} \equiv z / L_{z} \rightarrow 0$, then, there is a first transitional layer at distances from the probe of order of a local mean free path, $\lambda_{e 0} \sim \lambda_{e \infty} \times n_{\infty} / n_{0} \ll L_{z}$. Next, there is a collisionless layer where the ion Boltzmannlaw fails, at distances $z \sim R \ll \lambda_{e 0}\left(R / \lambda_{e \infty}\right.$ taken of order unity at most in Sec. II). Finally, quasineutrality itself fails in a sublayer embedded at $z \sim$ local $\lambda_{\text {De }}$. Multilayer structures in magnetized plasmas next to walls have been discussed in other respects as fully one dimensional. ${ }^{26-28}$

Throughout the inner layers, which are thin compared to the outer region, $z$-gradients are steep, and the electron continuity equation $\nabla \cdot n \bar{v}_{e}=0$ giving rise to (11) reads

$$
\frac{\partial}{\partial z} n v_{e z} \approx 0, \rightarrow n v_{e z} \equiv \int f_{e} v_{z} d \bar{v}=\mathrm{const}
$$

here we introduced the electron distribution function $f_{e}$. Similarly, the energy equation (3) for electrons, which gives rise to $\left(12^{\prime}\right)$, yields

$$
\begin{gathered}
\left(\frac{1}{2} m_{e} v_{e}^{2}+\frac{5}{2} k T_{e}-e \Phi\right) n v_{e z}+q_{e z}+\left(\overline{\bar{\Pi}}_{e} \cdot \bar{v}_{e}\right)_{z} \\
\equiv \int f_{e} v_{z} d \bar{v}\left(\frac{1}{2} m_{e} v^{2}-e \Phi\right)=\mathrm{const} ;
\end{gathered}
$$

the right-hand side of (3), or $\left(12^{\prime}\right)$, is clearly negligible throughout the inner region except for large $3 m_{e} R^{2} / m_{i} l_{e \infty}^{2}$, when (31b) will prove unnecessary. Two boundary conditions for $\tilde{z}=0, \tilde{r}<1$ can be obtained from Eqs. (31a) and (31b); in (31a), for instance, we need equating $\left.n v_{e z}\right|_{0}$, which may be written in terms of the outer solution, to the value of $\int f_{e} v_{z} d \bar{v}$ at the probe.

Note now that, for $\Phi_{0}>\Phi_{P}$ in the solution, electrons coming into the inner region move up an energy hill. In the limit $e\left(\Phi_{0}-\Phi_{P}\right) / k T_{e 0} \rightarrow \infty$, the probe, even though perfectly absorbing (no $v_{z}>0$ electrons at $z=0^{+}$), would act as a perfectly reflecting wall for the bulk plasma, with the electron current that reaches the probe from the outer region vanishingly small. Then, $f_{e}$ would be a Maxwell-Boltzmann (though truncated) distribution throughout the collisionless layer, and fully Maxwellian, but for a vanishingly small correction, in the outer region.

From the continuity of the solution on boundary conditions, we now expect that, for $e\left(\Phi_{0}-\Phi_{P}\right) / k T_{e 0}$ logarithmically large as found below, only the positive $v_{z}$-tail of the distribution function in the collisionless layer could differ to dominant order from the truncated Maxwell-Botzmann value. In the transitional layer we would have

$$
n / n_{0} \sim \exp \left[e\left(\Phi-\Phi_{0}\right) / k T_{e 0}\right]
$$

a result in conflict with Eq. $\left(5^{\prime}\right)$ (valid within this layer) unless $\Phi-\Phi_{0}$ vanishes there. Thus, the only dominant phenomenon occurring at $z \sim \lambda_{e 0}$ is the evolution of $f_{e}$ from a Maxwellian truncated at $v_{z}=\sqrt{ } 2 e\left(\Phi_{0}-\Phi_{P}\right) / m_{e}$ as $z / \lambda_{e 0}$ $\rightarrow 0$, to a full Maxwellian plus a small current-carrying distribution as $z / \lambda_{e 0} \rightarrow \infty$. The potential will increase from $\Phi_{P}$ at the probe to some (positive) value $\Phi_{0}$ over distances $z$ $\sim R$, and stay nearly constant at $z \sim \lambda_{e 0}$, only approaching zero in the outer region.

Using the Maxwell-Boltzmann distribution truncated at $v_{z}=0$ to evaluate the integrals in (31a) and (31b) at the probe we find

$$
\begin{aligned}
& \left.n v_{e z}\right|_{0}=-n_{0} \exp \left[-\frac{e\left(\Phi_{0}-\Phi_{P}\right)}{k T_{e 0}}\right] \times \frac{1}{4} \sqrt{\frac{8 k T_{e 0}}{\pi m_{e}}}, \quad\left(31 \mathrm{a}^{\prime}\right) \\
& \left(\frac{5}{2} k T_{e 0}-e \Phi_{0}\right) \times\left. n v_{e z}\right|_{0}+q_{e z 0}=\left.\left(2 k T_{e 0}-e \Phi_{P}\right) n v_{e z}\right|_{0},
\end{aligned}
$$

with

$$
n_{0}=n_{\infty} \exp \left(-Z_{i} e \Phi_{0} / k T_{i \infty}\right) .
$$

In writing the left-hand side of $\left(31 b^{\prime}\right)$ we used Eq. (3) in the approximation (12), valid in the outer region. Note that conservation of momentum flux in Eq. (2) throughout the inner region, will in general involve $\nabla \Phi$ (rather than $\Phi$ itself) through the term $n \partial \Phi / \partial z$; since $\nabla \Phi$ is unknown at the probe, no outer boundary condition would ensue. With $n v_{e z}$ and $q_{z}$ taken from $\left(5^{\prime}\right),(6)$, and (13), Eqs. $\left(31 \mathrm{a}^{\prime}\right)$ and $\left(31 \mathrm{~b}^{\prime}\right)$ provide two relations for the outer solution at $\tilde{z}=0, \tilde{r}<1$,

$$
\begin{aligned}
\widetilde{T}_{e 0}\left[\left.\left(\beta_{0}+1\right) \frac{\partial \widetilde{T}_{e}}{\partial \tilde{z}}\right|_{0}-\left.\frac{1}{2}\left(\widetilde{T}_{e 0}+\frac{\widetilde{T}_{i \infty}}{Z_{i}}\right) \frac{\partial \widetilde{\Phi}}{\partial \tilde{z}}\right|_{0}\right] \\
=\sqrt{\frac{\alpha_{0}}{2 \pi}} \frac{R}{l_{e \infty}} \exp \left[\frac{e \Phi_{P}}{k T_{e \infty}} \frac{1}{\widetilde{T}_{e 0}}-\left(\widetilde{T}_{e 0}+\frac{\widetilde{T}_{i \infty}}{Z_{i}}\right) \frac{\widetilde{\Phi}_{0}}{2 \widetilde{T}_{e 0}}\right],
\end{aligned}
$$




$$
\begin{gathered}
{\left[\left.\left(\beta_{0}+1\right) \frac{\partial \widetilde{T}_{e}}{\partial \widetilde{z}}\right|_{0}-\left.\frac{1}{2}\left(\widetilde{T}_{e 0}+\frac{\widetilde{T}_{i \infty}}{Z_{i}}\right) \frac{\partial \widetilde{\Phi}}{\partial \tilde{z}}\right|_{0}\right]\left[\beta_{0}+\frac{1}{2}\right.} \\
\left.+\frac{1}{\widetilde{T}_{e 0}}\left(\frac{e \Phi_{P}}{k T_{e \infty}}-\frac{\widetilde{T}_{i \infty} \widetilde{\Phi}_{0}}{2 Z_{i}}\right)\right]=-\left.\alpha_{0} \gamma_{0} \frac{\partial \widetilde{T}_{e}}{\partial \tilde{z}}\right|_{0}
\end{gathered}
$$

If boundary condition (33b) were just ignored and $T_{e}$ taken as uniform, one would find $\partial \widetilde{\Phi} /\left.\partial \tilde{z}\right|_{0}<0$ in (33a), making $\Phi_{0}$ a maximum of the potential; this hill value, both positive and larger than $\Phi_{P}$ for a range of probe bias, was the potential overshoot first noticed in Ref. 7. However, when both (33a) and (33b) are taken into account, and $\Phi_{P}$ is negative enough, one finds $\partial \widetilde{\Phi} /\left.\partial \widetilde{z}\right|_{0}$ as well as $\partial \widetilde{T}_{e} /\left.\partial \tilde{z}\right|_{0}$ positive; this means that the potential hill lies well inside the outer region. Note next that, for $e \Phi_{P} / k T_{e \infty}$ negative enough at fixed $R / l_{e \infty}$, both $\Phi$ and $\widetilde{T}_{e}-1$ vanish with $R / l_{e \infty}$ $\times e \Phi_{P} / k T_{e \infty} \times \exp \left(e \Phi_{P} / k T_{e \infty}\right)$. Finally, write the right-hand side of (33a), with the large factor $R / l_{e^{\infty}}$, as

$$
\begin{aligned}
\text { RHS of Eq. (33a) } \equiv & \sqrt{\frac{\alpha_{0}}{2 \pi}} \exp \left[\ln \frac{R}{l_{e \infty}}-\frac{Z_{i} e \Phi_{0}}{k T_{i \infty}}\right. \\
& \left.-\frac{e\left(\Phi_{0}-\Phi_{P}\right)}{k T_{e 0}}\right],
\end{aligned}
$$

now using dimensional variables for convenience of discussion. Clearly, for fixed $e \Phi_{P} / k T_{e \infty}$, and $R / l_{e \infty}$ large enough, $\Phi_{0}$ will vary as $\ln \left(R / l_{e^{\infty}}\right)$; in order to satisfy (33a) the bracket in (34) must vanish to order $\ln \left(R / l_{e^{\infty}}\right)$, a condition rewritten as

$$
\frac{e\left(\Phi_{0}-\Phi_{P}\right)}{k T_{e 0}}-\frac{T_{i \infty}}{T_{i \infty}+Z_{i} T_{e 0}}\left[\ln \frac{R}{l_{e \infty}}-\frac{Z_{i} e \Phi_{P}}{k T_{i \infty}}\right]=O(1)
$$

and, using (32), as

$$
\left[1+\frac{T_{i \infty}}{Z_{i} T_{e 0}}\left(1-\frac{\Phi_{P}}{\Phi_{0}}\right)\right] \ln \frac{n_{\infty}}{n_{0}}-\ln \frac{R}{l_{e \infty}}=O(1) .
$$

Equations (35) and (35') show $e\left(\Phi_{0}-\Phi_{P}\right) / k T_{e 0}$ to be logarithmically large, and $L_{z} / \lambda_{e 0} \sim\left(R / l_{e \infty}\right) \times n_{0} / n_{\infty}$, to be large, as advanced.

Equation (35) also suggests $\Phi_{0}-\Phi_{P}$ will decrease as $\Phi_{P}$ increases, with $\Phi_{P}$ overtaking $\Phi_{0}$ at

$$
\frac{Z_{i} e \Phi_{P}}{k T_{i \infty}}-\ln \frac{R}{l_{e \infty}}=O(1),
$$

where, certainly, $e\left(\Phi_{0}-\Phi_{P}\right) / k T_{e 0}$ is not logarithmically large. Note also that for $\Phi_{0}=\Phi_{P}$, Eq. (35') gives $n_{\infty} / n_{0}$ $\sim R / l_{e \infty}$, or $\lambda_{e 0} \sim L_{z}$. Our analysis, therefore, breaks down on both counts before overtaking occurs. We might reasonably use our results right up to overtaking but not beyond it.

\section{DISCUSSION OF RESULTS}

Boundary conditions (33a) and (33b) introduce dimensionless parameters $e \Phi_{P} / k T_{e \infty}$ and $R / l_{e \infty}$, in addition to $T_{i \infty} / T_{e \infty}, Z_{i}$, and $3 m_{e} R^{2} / m_{i} l_{e \infty}^{2}\left(\sim R^{2} / l_{i \infty}^{2}\right)$, appearing in Eqs. (11) and $\left(12^{\prime}\right)$. The Debye length does not enter the analysis. The undisturbed mean free path $\lambda_{e \infty}$ determines the

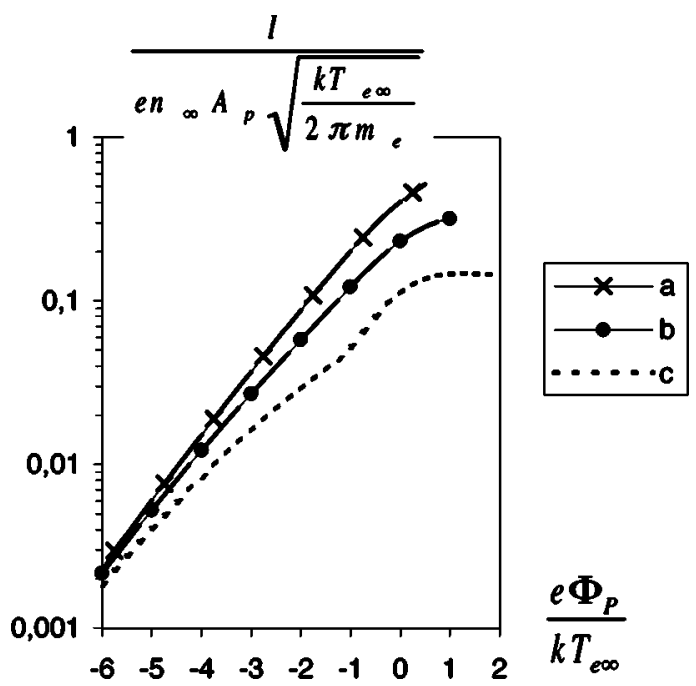

FIG. 1. Electron current to the probe normalized with the undisturbed random current to a two-sided projected area $A_{P}=2 \pi R^{2}$. Values of dimensionless parameters are $Z_{i}=T_{i \infty} / T_{e \infty}=1,3 m_{e} R^{2} / m_{i} l_{e \infty}^{2}=0$, and $R / l_{e \infty}=4$ (line a), 10 (b), and 25 (c)

spatial extent of the perturbations $\left(L_{z} \sim \lambda_{e \infty} R / l_{e \infty}\right)$ but disappears from the results. Our analysis did impose conditions $\lambda_{e \infty} / l_{e \infty} \geqslant O\left(\sqrt{ } m_{i} / m_{e}\right)$, for easy handling of ion transport in model validation, and $\lambda_{e \infty} / R \geqslant O(1)$, for a general separation of the transitional and collisionless layers lying next to the probe.

Condition (27) on probe size allows arbitrary values of $3 m_{e} R^{2} / m_{i} l_{e \infty}^{2}$. If this parameter is large, Eq. $\left(12^{\prime}\right)$ just yields $T_{e}(z, r)=T_{i \infty}$ (electrons keep isothermal throughout collection), also implying $T_{e \infty}=T_{i \infty}$ [the large collection length $L_{z}$ in (9) requires a plasma so extensive that collisions make for equal undisturbed temperatures]. For $3 m_{e} R^{2} / m_{i} l_{e \infty}^{2}$ of order unity collection is nonisothermal but $T_{e \infty}$ and $T_{i \infty}$ need still be equal. Nonisothermal collection with $T_{i \infty} / T_{e \infty}$ arbitrary corresponds to $3 m_{e} R^{2} / m_{i} l_{e \infty}^{2}$ negligibly small.

Clearly, the analysis of Ref. 7 is only valid for the large $3 m_{e} R^{2} / m_{i} l_{e \infty}^{2}$ regime. Note that, with $\widetilde{T}_{e}(\tilde{z}, \tilde{r}) \equiv 1$ in (33a), parameters $e \Phi_{P} / k T_{e \infty}$ and $R / l_{e \infty}$ enter the solution for this regime in the single combination $e \Phi_{P} / k T_{e \infty}+\ln \left(R / l_{e \infty}\right)$. That solution will naturally not satisfy condition (33b), as a result of our having dropped all derivatives in the right-hand side of $\left(12^{\prime}\right)$. This irrelevant boundary-layer effect is clarified in the Appendix by solving the general linear problem obtained at large and negative $e \Phi_{P} / k T_{e \infty}$, and then taking the limit $3 m_{e} R^{2} / m_{i} l_{e \infty}^{2} \rightarrow \infty$.

Figures 1-6 show current $I$, and potential $\Phi_{0}$ and electron temperature $T_{e 0}$ "at the probe" averaged over its cross section,

$$
\begin{aligned}
& I=2 \int_{0}^{R} 2 \pi r d r \times\left(-\left.e n v_{e z}\right|_{0}\right), \\
& \left\langle\Phi_{0}\right\rangle \equiv \int_{0}^{R} \Phi_{0} 2 r d r / R^{2},\left\langle T_{e 0}\right\rangle \equiv \int_{0}^{R} T_{e 0} 2 r d r / R^{2},
\end{aligned}
$$

up to the bias yielding $\left\langle\Phi_{0}\right\rangle=\Phi_{P}$. In Figs. 1-3, we set $Z_{i}=T_{i \infty} / T_{e \infty}=1$, and $3 m_{e} R^{2} / m_{i} l_{e \infty}^{2} \approx 0$, and took moder- 


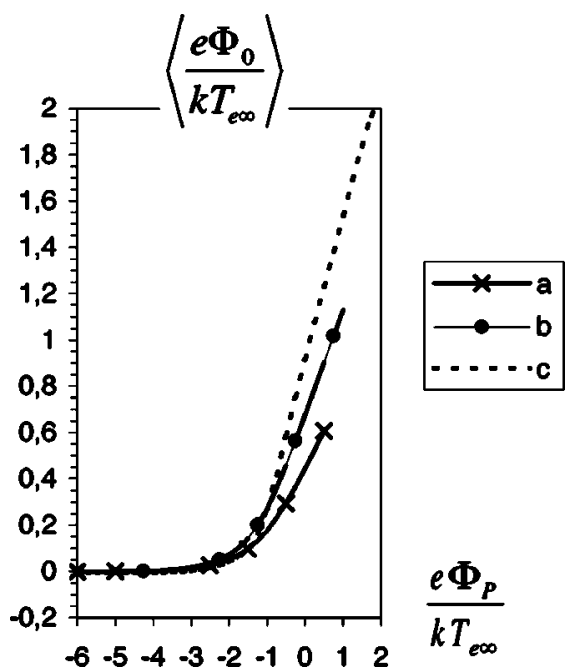

FIG. 2. Normalized potential at the transition between outer and inner regions averaged over the probe cross section. Dimensionless parameters as in Fig. 1.

ately large values of $R / l_{e \infty}: 4,10$, and 25 . In Figs. 4-6 we took $Z_{i}=1, R / l_{e \infty}=25$, with $T_{i \infty} / T_{e \infty}=1$, and $3 m_{e} R^{2} / m_{i} l_{e \infty}^{2}$ $=0,1$ (1.02 for $H$ ions), and $\infty$ (here the formal limit that corresponds to setting $T_{e} \equiv T_{e \infty}$ ). Also shown in Figs. 4-6 are results for $T_{i \infty} / T_{e \infty}=0.3$ and $3 m_{e} R^{2} / m_{i} l_{e \infty}^{2}=0$. We note that low $T_{i \infty} / T_{e \infty}$ and high $Z_{i}$ effects are roughly similar.

Our results show cooling at negative bias that is stronger the smaller $3 m_{e} R^{2} / m_{i} l_{e \infty}^{2}$ but also the larger $R / l_{e \infty}$; a minimum of $\left\langle T_{e 0}>\right.$ occurs at $e \Phi_{P} \approx-k T_{e \infty}$, with heating setting in around zero bias. At low $3 m_{e} R^{2} / m_{i} l_{e \infty}^{2}$, the potential $\left\langle\Phi_{0}\right\rangle$ is very small in the bias range to the left of the $<T_{e 0}>$ minimum, when the potential overshoot occurs far away from the probe. Current reduction is again stronger the larger $R / l_{e \infty}$ but also the smaller $3 m_{e} R^{2} / m_{i} l_{e \infty}^{2}$, although this last parameter has no sensible effect on the "saturation" current at positive bias. Note that, at a fixed mass ratio

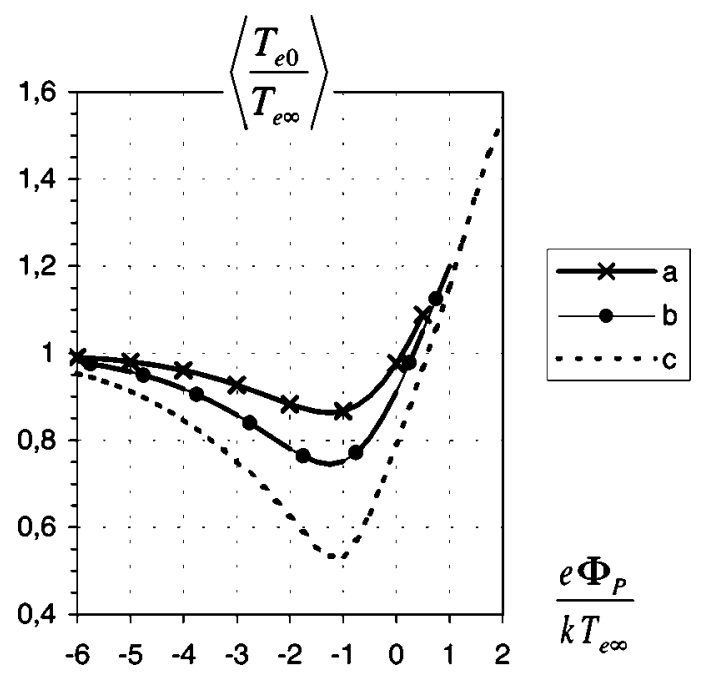

FIG. 3. Normalized electron temperature at the transition between outer and inner regions, averaged over the probe cross section. Dimensionless parameters as in Fig. 1.

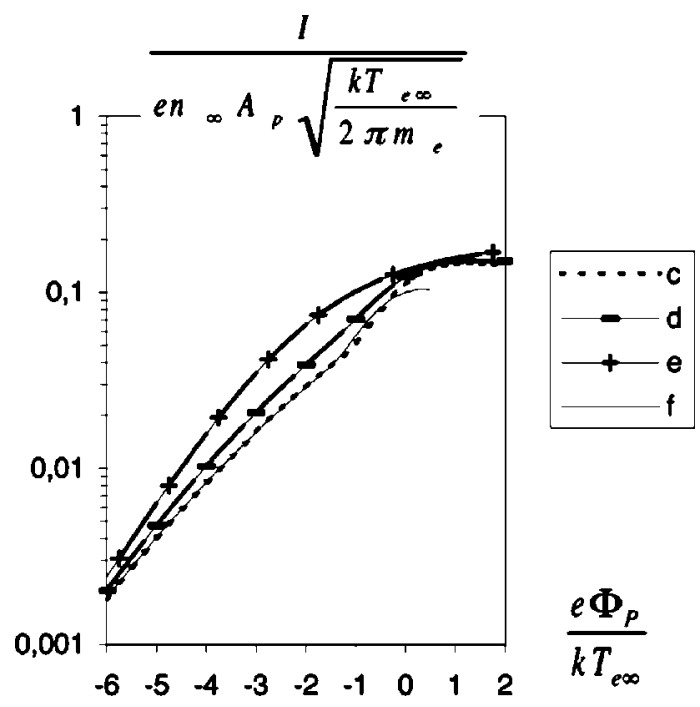

FIG. 4. Same as in Fig. 1. Values of dimensionless parameters: $Z_{i}=1$, $R / l_{e \infty}=25$, with $T_{i \infty} / T_{e \infty}=1$ and $3 m_{e} R^{2} / m_{i} l_{e \infty}^{2}=0$ (c), 1 (d), and $\infty$ (e); or $T_{i \infty} / T_{e \infty}=0.3$, and $3 m_{e} R^{2} / m_{i} l_{e \infty}^{2}=0$ (f).

$m_{e} / m_{i}$, cooling and current reduction would be greatest at some large, intermediate value of the ratio $R / l_{e^{\infty}}$.

The decrease of current $I$ with the parameter $3 m_{e} R^{2} / m_{i} l_{e \infty}^{2}$ (Fig.4) relates to the cooling. Ignoring the variations of $\Phi_{0}$ and $T_{e 0}$ across the probe cross section one may use Eq. $\left(31 a^{\prime}\right)$ to write the approximate relation

$$
\ln I \approx \frac{1}{2} \ln \frac{T_{e 0}}{T_{e \infty}}-\frac{e\left(\Phi_{0}-\Phi_{P}\right)}{k T_{e 0}}-\frac{e \Phi_{0}}{k T_{e \infty}}+\text { const, }
$$

where $I$ decreases with both increasing $\Phi_{0}$ or decreasing $T_{e 0}$. As one reduces $3 m_{e} R^{2} / m_{i} l_{e \infty}^{2}$ from the large values corresponding to results in Ref. 7, the effect a $\Phi_{0}$ decrease (Fig. 5 ) has on the current is more than balanced by stronger cooling (Fig. 6).

Next, note that the slope in the $\ln I-\Phi_{P}$ graph can be obtained from Eq. (37),

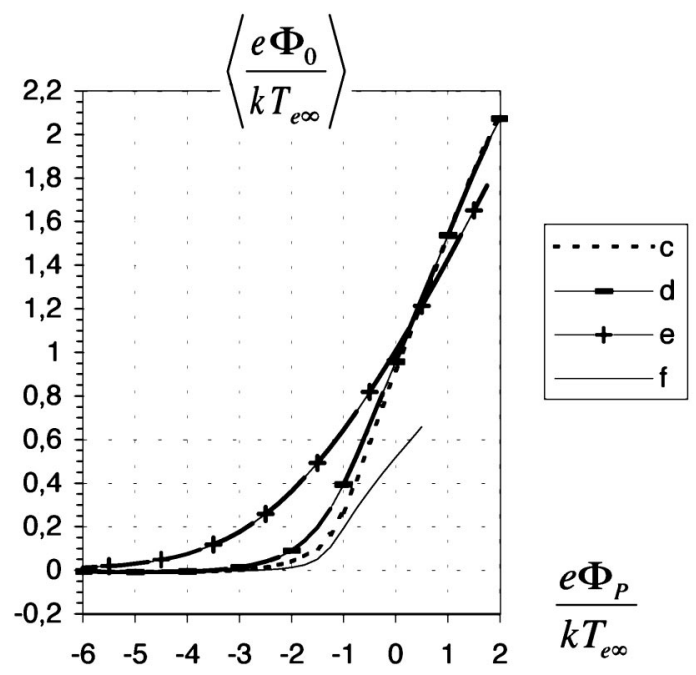

FIG. 5. Same as in Fig. 2. Dimensionless parameters as in Fig. 4. 


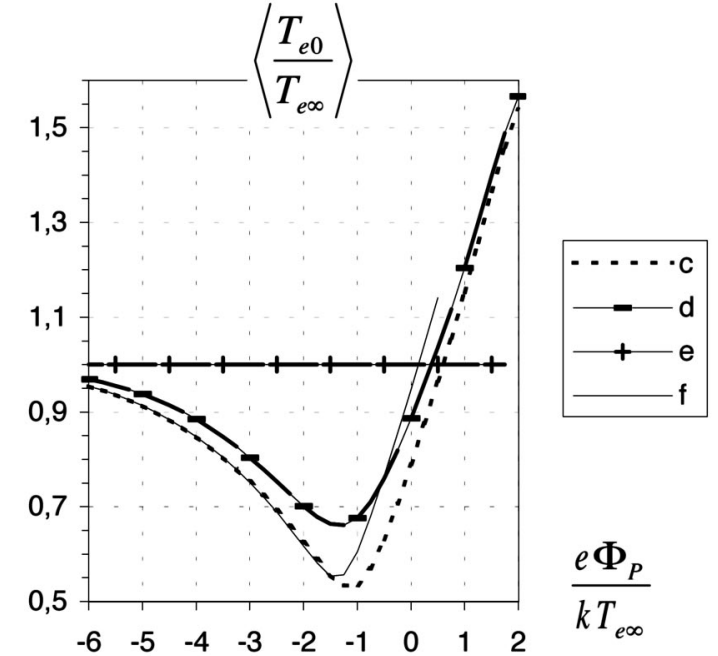

FIG. 6. Same as in Fig. 3. Dimensionless parameters as in Fig. 4.

$$
\begin{aligned}
\frac{d \ln I}{d \Phi_{P}} \approx & {\left[1-\left(1+\frac{T_{e 0}}{T_{e \infty}}\right) \frac{d \Phi_{0}}{d \Phi_{P}}\right] \frac{e}{k T_{e 0}} } \\
& +\left[\frac{1}{2}+\frac{e\left(\Phi_{0}-\Phi_{P}\right)}{k T_{e 0}}\right] \frac{1}{T_{e 0}} \frac{d T_{e 0}}{d \Phi_{P}}
\end{aligned}
$$

The slope $d \ln I / d \Phi_{P} \approx e / k T_{e \infty}$ at $\Phi_{P}$ negative enough (with $\Phi_{0} \approx 0, T_{e 0} \approx T_{e \infty}$ ) is inversely proportional to the unperturbed electron temperature, as in unmagnetized plasmas. At higher bias, however, one might expect that cooling would result in the slope increasing with decreasing $3 m_{e} R^{2} / m_{i} l_{e^{\infty}}^{2}$ but Fig. 4 shows otherwise. This clearly arises from the fact that cooling is $\Phi_{P}$-dependent, the last term in (38) being negative to the left of the $T_{e 0}$ minimum. For intense cooling (large $R / l_{e \infty}$ ), the slope tilts upward as $\Phi_{P}$ increases beyond that minimum, producing a $\ln I$ vs $\Phi_{P}$ profile that is concave upward (Figs. 1, 4), a feature found in some experiments ${ }^{29}$ and displayed in unmagnetized, two electron-temperature plasmas, too.

The potential overshoot in the magnetic shadow of a probe was noticed in Ref. 7 through a careful analysis of transport in collisional, fully ionized plasmas; the first experimental evidence of overshoot, however, was found by Kawaguchi and Tanaka ${ }^{8}$ in weakly ionized plasmas, after Nigoyi and Cohen had shown that the same overshoot existed under weak ionization. ${ }^{8}$ We now suggest that the new features discussed above may similarly be present under other transport laws. Anomalous, turbulent transport such as Bohm diffusion would mainly produce a shadow characteristic length much smaller than $L_{z}$ as given with Eq. (9), but $L_{z}$ does not figure in the results. Replacing Eqs. (7)-(8) for the radial flux by Bohm's Law (with its different dependence on $\Omega_{e} \tau_{e} \propto T_{e}^{3 / 2} / n$ ) would also modify Eq. (11), but should only affect results to some quantitative degree. Nonetheless, uncertainties in modeling the energy equation, while keeping full consistency in the overall model, make the extension of our results to turbulent transport a difficult though challenging task.

\section{ACKNOWLEDGMENTS}

This work was supported by the Comisión Interministerial de Ciencia y Tecnología of Spain under Grant No. PB97-0574-C04-1.

\section{APPENDIX: HIGHLY NEGATIVE PROBE BIAS}

As noted in Sec. V, both $\widetilde{\Phi}$ and $\widetilde{T}_{e}-1 \equiv \delta \widetilde{T}_{e}$ are small at logarithmically large and negative $e \Phi_{P} / k T_{e^{\infty}}$. We may then linearize Eqs. (11), (12') and boundary conditions (33a), (33b) and introduce Hankel integral transforms,

$$
\begin{aligned}
& \widetilde{\Phi}_{q}(\tilde{z}) \equiv \int_{0}^{\infty} \widetilde{\Phi}(\tilde{r}, \tilde{z}) \tilde{r} J_{0}(q \tilde{r}) d \tilde{r}, \\
& \widetilde{\Phi}(\tilde{r}, \tilde{z})=\int_{0}^{\infty} \widetilde{\Phi}_{q}(\tilde{z}) q J_{0}(q \tilde{r}) d q,
\end{aligned}
$$

with similar expressions for $\delta \widetilde{T}_{e}$; here, $J_{0}$ is the Bessel function of the first kind and zero order. Boundary conditions at $\tilde{r}=0, \tilde{r} \rightarrow \infty$ are then automatically satisfied.

Equations (11) and $\left(12^{\prime}\right)$ with $\widetilde{T}_{i \infty}=1$ now become

$$
\begin{aligned}
& \frac{Z_{i}+1}{Z_{i}}\left(\frac{d^{2}}{d \widetilde{z}^{2}}-q^{2}\right) \widetilde{\Phi}_{q}-\left[2\left(1+\beta_{0}\right) \frac{d^{2}}{d \widetilde{z}^{2}}+q^{2}\right] \delta \widetilde{T}_{\mathrm{eq}}=0, \\
& \frac{Z_{i}+1}{Z_{i}}\left[\left(\frac{5}{2}+\beta_{0}\right) \frac{d^{2}}{d \widetilde{z}^{2}}-q^{2}\right] \widetilde{\Phi}_{q} \\
& -\left[2\left(\alpha_{0} \gamma_{0}+\frac{5}{2}+\frac{7}{2} \beta_{0}+\beta_{0}^{2}\right) \frac{d^{2}}{d \widetilde{z}^{2}}+\left(\frac{11}{2}-2 \gamma_{1}^{\prime}\right)\right] \delta \widetilde{T}_{\mathrm{eq}} \\
& =-\frac{3 m_{e} R^{2}}{m_{i} l_{e \infty}^{2}} \delta \widetilde{T}_{\mathrm{eq}} .
\end{aligned}
$$

The characteristic equation for system (A2), (A3) has two negative roots, $-\eta_{1}(q)$ and $-\eta_{2}(q)$, and two positive roots. Using the boundary conditions for $\tilde{z} \rightarrow \infty$, we may write

$$
\begin{aligned}
& \widetilde{\Phi}_{q}=A_{1}(q) \exp \left(-\eta_{1} \tilde{z}\right)+A_{2}(q) \exp \left(-\eta_{2} \tilde{z}\right), \\
& \delta \widetilde{T}_{\mathrm{eq}}=B_{1}(q) \exp \left(-\eta_{1} \tilde{z}\right)+B_{2}(q) \exp \left(-\eta_{2} \tilde{z}\right), \\
& \frac{B_{j}}{A_{j}}=\frac{Z_{i}+1}{Z_{i}} \frac{\eta_{j}^{2}-q^{2}}{2\left(1+\beta_{0}\right) \eta_{j}^{2}+q^{2}}, \quad j=1,2 .
\end{aligned}
$$

Finally, using $\widetilde{\Phi}(\tilde{r}, \tilde{z})$ given by (A1b) and the corresponding expression for $\delta \widetilde{T}_{e}(\tilde{r}, \tilde{z})$ in the remaining boundary conditions, (30a), (30b) and (33a), (33b), one finds two dual integral equations,

$$
\begin{aligned}
& \int_{0}^{\infty}\left(\eta_{1} A_{1}+\eta_{2} A_{2}\right) q J_{0}(q \tilde{r}) d q=a \text { for } \tilde{r}<1, \\
& (=0 \text { for } \tilde{r}>1), \\
& \int_{0}^{\infty}\left(\eta_{1} B_{1}+\eta_{2} B_{2}\right) q J_{0}(q \tilde{r}) d q=b \text { for } \tilde{r}<1, \\
& (=0 \text { for } \tilde{r}>1) .
\end{aligned}
$$


Here,

$$
\begin{aligned}
a= & \sqrt{\frac{\alpha_{0}}{2 \pi}} \frac{R}{l_{e \infty}}\left[1+\frac{1+\beta_{0}}{\alpha_{0} \gamma_{o}}\left(\frac{e \Phi_{P}}{k T_{e \infty}}+\beta_{0}+\frac{1}{2}\right)\right] \frac{2 Z_{i}}{1+Z_{i}} \\
& \times \exp \left(\frac{e \Phi_{P}}{k T_{e \infty}}\right), \\
b= & \sqrt{\frac{\alpha_{0}}{2 \pi}} \frac{R}{l_{e \infty}}\left(\frac{e \Phi_{P}}{k T_{e \infty}}+\beta_{0}+\frac{1}{2}\right) \frac{\exp \left(e \Phi_{P} / k T_{e \infty}\right)}{\alpha_{0} \gamma_{0}}
\end{aligned}
$$

where we retained logarithmically small terms. The solution to Eqs. (A5) and (A6) is

$$
\begin{aligned}
& \eta_{1} A_{1}+\eta_{2} A_{2}=a J_{1}(q) / q, \\
& \eta_{1} B_{1}+\eta_{2} B_{2}=b J_{1}(q) / q,
\end{aligned}
$$

$J_{1}$ being the Bessel function of first order. Equations (A4a), (A4b) and (A7a), (A7b) determine $A_{1}(q), A_{2}(q), B_{1}(q)$, and $B_{2}(q)$, allowing for a full and explicit analytical solution. In particular, one obtains the average potential and temperature "at the probe," in addition to the current, in terms of definite $q$-integrals.

For $3 m_{e} R^{2} / m_{i} l_{e \infty}^{2} \equiv 1 / \epsilon$ large, we find

$$
\begin{gathered}
\eta_{1}=O(1), \quad \eta_{2}=O(1 / \sqrt{ } \epsilon), A_{1}=O(1), \\
A_{2}=O\left({ }_{\sqrt{ }} \epsilon\right), \quad B_{1}=O(\epsilon) \rightarrow B_{2} \eta_{2} \approx b J_{1}(q) / q .
\end{gathered}
$$

Using these results in the inverse transform for $\delta \widetilde{T}_{\mathrm{eq}}$,

$$
\begin{aligned}
\widetilde{T}_{e}(\tilde{r}, \tilde{z})-1= & \int_{0}^{\infty}\left[B_{1} \exp \left(-\eta_{1} \tilde{z}\right)\right. \\
& \left.+B_{2} \exp \left(-\eta_{2} \tilde{z}\right)\right] q J_{0}(q \tilde{r}) d q,
\end{aligned}
$$

we find $\widetilde{T}_{e}(\tilde{r}, \tilde{z}) \rightarrow 1$ as $3 m_{e} R^{2} / m_{i} l_{e \infty}^{2} \rightarrow \infty$, but

$$
-\left.\frac{\partial \widetilde{T}_{e}}{\partial \tilde{z}}\right|_{0} \rightarrow \int_{0}^{\infty} \eta_{2} B_{2} q J_{0}(q \tilde{r}) d q=b \neq 0
$$

which is the boundary condition on the temperature gradient resulting from (33a) and (33b).
${ }^{1}$ L. W. Parker and B. L. Murphy, J. Geophys. Res. 72, 1631 (1967).

${ }^{2}$ J. Rubinstein and J. G. Laframboise, Phys. Fluids 25, 1174 (1982); L. J. Sonmor and J. G. Laframboise, Phys. Fluids B 3, 2472 (1991).

${ }^{3}$ N. Singh, B. I. Vashi, and L. C. Leung, Geophys. Res. Lett. 21, 833 (1994).

${ }^{4}$ L. M. Linson, J. Geophys. Res. 74, 2368 (1969).

${ }^{5}$ I. H. Hutchinson, Phys. Fluids 30, 3777 (1987).

${ }^{6}$ K. Günther, Contrib. Plasma Phys. 30, 51 (1990).

${ }^{7}$ J. R. Sanmartín, Phys. Fluids 13, 103 (1970).

${ }^{8}$ K. K. Nigoyi and I. M. Cohen, Phys. Fluids 16, 69 (1973); M. Kawaguchi and H. Tanaka, ibid. 24, 2378 (1981).

${ }^{9}$ D. Bohm, in The Characteristics of Electrical Discharges in Magnetic Fields, edited by A.Guthrie and R.K.Wakerling (McGraw-Hill, New York, 1949), Chap. 3; M. Sugawara, Phys. Fluids 9, 797 (1966).

${ }^{10}$ J. G. Laframboise and L. J. Sonmor, J. Geophys. Res. 98, 337 (1993).

${ }^{11}$ J. R. Sanmartin and R. D. Estes, Phys. Plasmas 6, 395 (1999).

${ }^{12} \mathrm{~W}$. B. Thomson, "Preliminary investigation of the electrodynamics of a conducting tether," in Spacecraft Environmental Interactions Technology, edited by C. K. Purvis and C. P. Pike, Rep. AFGL-TR-85-0018/NASA Conf. Publ. 2359 (National Aeronautics and Space Adminitration, Washington, D.C., 1985), pp. 649-662.

${ }^{13}$ N. Singh and V. S. Chaganti, J. Geophys. Res. 99, 469 (1994); N. Singh, L. C. Leung, and B. I. Vashi, ibid. 102, 195 (1997).

${ }^{14}$ J. G. Laframboise, J. Geophys. Res. 102, 2417 (1997); G. Vannaroni, M. Dobrowolny, J. P. Lebreton, et al., Geophys. Res. Lett. 25, 749 (1998); D. L. Cooke and I. Katz, ibid. 25, 753 (1998).

${ }^{15}$ A. C. Tribble, J. S. Pickett, N. D’Angelo, and G. B. Murphy, Planet. Space Sci. 37, 1001 (1989).

${ }^{16}$ I. Katz, E. Melchioni, M. Mandell, et al. J. Geophys. Res. 99, 8961 (1994).

${ }^{17}$ J. E. Sorensen, N. H. Stone, and K. H. Wright, Jr., J. Geophys. Res. 102, 24117 (1997).

${ }^{18}$ P. C. Stangeby, in Plasma Diagnostics. Surface Analysis and Interactions, edited by O. Auciello and D. L. Flamm (Academic, San Diego, 1989), p. 157.

${ }^{19}$ K. Chung and R. D. Bengtson, Phys. Plasmas 4, 2928 (1997).

${ }^{20}$ M. Weinlich and A. Carlson, Phys. Plasmas 4, 2151 (1997).

${ }^{21}$ J. P. Gunn, Phys. Plasmas 4, 4435 (1997).

${ }^{22}$ S. I. Braginskii, in Reviews of Plasma Physics, edited by M. A. Leontovich (Consultants Bureau, New York, 1965), Vol. 1, p. 205.

${ }^{23}$ E. Lifchitz and L. Pitayevski, Cinétique Physique (Mir, Moscow, 1990), Chap. V.

${ }^{24}$ E. M. Epperlein and M. G. Haines, Phys. Fluids 29, 1029 (1986).

${ }^{25}$ J. R. Sanmartín, in Nuclear Fusion by Inertial Confinement, edited by G. Velarde, Y. Ronen, and J. M. Martínez-Val (Chemical Rubber, Boca Raton, 1993), Chap. 7.

${ }^{26}$ R. Chodura, Phys. Fluids 25, 1628 (1982).

${ }^{27}$ K. U. Riemann, Phys. Plasmas 1, 552 (1994).

${ }^{28}$ E. Ahedo, Phys. Plasmas 4, 4419 (1997); 6, 4200 (1999).

${ }^{29}$ K. Guenther, A. Herrman, M. Laux, P. Pech, and H.-D. Reiner, J. Nucl. Mater. 176\&177, 236 (1990). 